\title{
Differential Potential Distribution Observation in Transmission Electron Microscope with Conventional Thermal Electron Gun
}

\author{
Katsuhiro Sasaki ${ }^{1}$, Hirokazu Sasaki ${ }^{2}$ and Sohei Saito ${ }^{1}$ \\ 1. Chemical Analysis Research Section, R\&D Division, UACJ Company, Nagoya, Japan \\ 2. Advanced Technologies R\&D Laboratories, Furukawa Electric Co. Ltd., Yokohama, Japan
}

The potential distribution in a material, particularly in semiconductors, has been imaged and measured by using electron holography [1], Lorentz microscopy [2] in a transmission electron microscope and DPC method in a scanning transmission electron microscope [3]. However, all these methods require the dedicated specially designed microscope or limited imaging condition. We have been developing a method to visualize the electric filed in a conventional electron microscope with a thermal electron gun $[4,5]$. We have modified our method applicable for built in potential in a material.

The specimen used in this work is a pn junction in GaAs with the dopant concentration of $10^{-19}$ [6]. The cross-sectional transmission electron microscopy (TEM) specimen was prepared using focused ion beam (FIB) apparatus SIINT SMI3050TB and the FIB damaged layers were well removed using Ar ion beam [7]. TEM observation was performed in JEM-2010 with the accelerating voltage of $200 \mathrm{kV}$. The specimen was observed in the imaging mode in which the objective lens was turned off and imaged by the objective mini (OM) lens. The largest size of selected area diffraction (SAD) aperture was used to obtain pseudo liner shape of the shadow aperture [5]. The specimen was carefully tilted to avoid the diffraction contrast as in the case of electron holography.

In the imaging mode named "Low Mag" used in this work, the SAD aperture i.e., the shadow aperture, is located between the OM lens and the image plane of the lens as shown in Fig. 1. When the electron beam has certain divergency, the shadow of the aperture edge has diffused contrast, however, image of the specimen does not be affected. The intensity of electron beam focused on the image plane is determined by the ration of obstructed electrons in the diverged electron beam on the aperture plane as indicated by the lines 1,2 , and 3 . The observed image and intensity profile of the edge of the shadow aperture without the specimen is shown in Fig. 2. If the shift of diverged electron beam on the aperture plane as the result of deflection of the beam due to the potential difference in the specimen, it caused the deviation of the intensity from the gradual change shown in the lower part of Fig. 2.

Figure 3 shows the images of GaAs specimen in which the pn junction aligned parallel to the electron beam and the junction runs vertically in each image. The specimen thickness has been estimated to be $190 \mathrm{~nm}$ from the electron hologram obtained separately in the other work. In Fig. 3, the left side of the images correspond to the original surface of the cross-sectional specimen and coated by the $\mathrm{C}$ deposition layer indicated by "Deposition" and $\mathrm{p}$ and $\mathrm{n}$ layers are positioned from left side under the "Deposition" layer as indicated by " $p$ " and " $n$ ". Figure $3 b$ shows the ordinarily bright filed image in which the position of the pn junction is not imaged. In the images $3 \mathrm{a}$ and $3 \mathrm{c}$, the diffuse contrast of the aperture edge running parallel to the pn junction is overlapped in the middle of the specimen image. The aperture is positioned left and right side in Fig. $3 \mathrm{a}$ and 3c, respectively. The dark and bright lines are observed in originally continuous contrast change of the aperture edge, which is deduced to be caused by the deflection of the electron beam at the pn junction in the specimen. In the specimen, the electron beam is deflected from $n$-side to $\mathrm{p}$-side, which will cause the difference of the obstruction ration of the electron 
beam. In our case, electron beam will deflect to left side. As the result, the obstruction ration of the electron beam increase and decrease when the aperture positioned left and right side, as illustrated in the insets of Fig. 3, which produce the dark and bright lines, respectively. The thickness of the pn junction estimated by the thickness of the dark and bright lines was $46 \mathrm{~nm}$ which showd slightly thicker value obtained from the dopant profile measured with SIMS, however, good correspondence to the electron holography measurement [8].

The differential potential distribution in the semiconductor at a pn junction was successfully imaged in a conventional TEM equipped with a conventional thermal electron gun. The quantitative value of potential distribution could be obtained from the measured value.

References:

[1] A. Tonomura, Reviews of Modern Physics 59 (1987) p. 639.

[2] J. N. Chapman, J. Phys. D 17 (1984) p. 623.

[3] N. Shabata et al, Scientific Reports 5 (2015), p. 10040.

[4] K. Sasaki, and H. Saka, Materials Science Forum 475-479 (2005) p. 4029.

[5] K. Sasaki et al, in "The Transmission Electron Microscope", ed. M. Khan, (InTech, Rijeka) p.1.

[6] H. Sasaki et al, Microscopy 63 (2014), p. 235.

[7] H. Takahashi et al, Proc. $16^{\text {th }}$ Int. Microscopy Congress (2006), p.1104

[8] The authors thank to Dr. Y Hori for his SIMS and electron holography measurements.

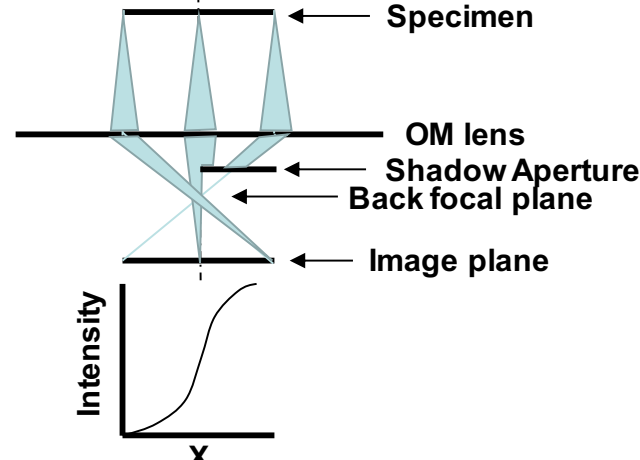

Figure 1. Geometrical optics and intensity profile of the aperture edge.
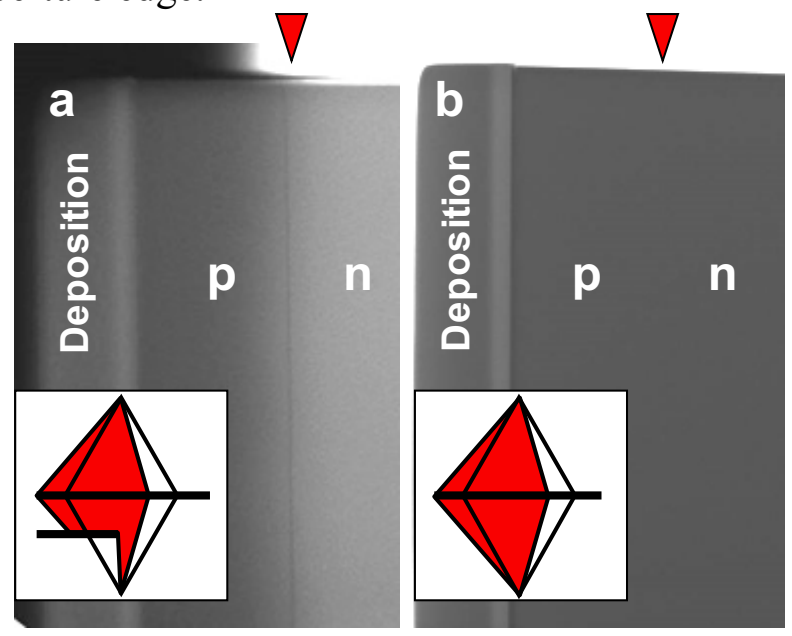

Figure 2. The observed image and intensity profile of the aperture edge

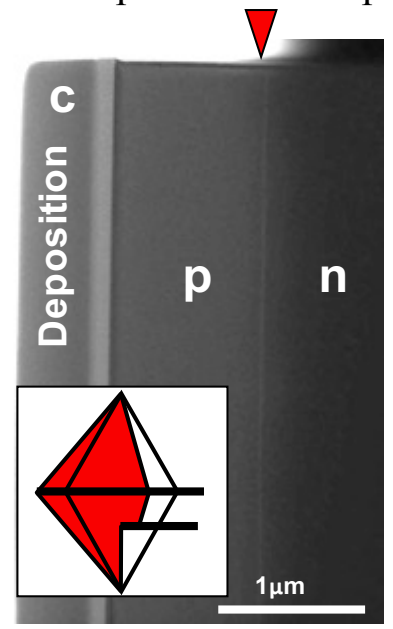

Figure 3. BF image of pn junction with the aperture in (a) left, (b) without, (c) right hand side. Insets show the geometrical optics of the deflected beam with the aperture, schematically. 